\title{
Effect of accelerated aging treatment on a surface property and dynamic mechanical properties of commercial wood-based panels
}

\author{
Sahriyanti Saad ${ }^{1,2} \cdot$ Hikaru Kobori $^{1} \cdot$ Yoichi Kojima $^{1} \cdot$ Shigehiko Suzuki $^{1}$
}

Received: 21 March 2017/ Accepted: 28 May 2017/Published online: 6 July 2017

(C) The Japan Wood Research Society 2017

\begin{abstract}
Changes in surface property and mechanical properties in commercial particleboards and medium density fiberboards subjected to repetitive relative humidity treatment (i.e., aging treatment) were investigated. The stylus technique was used to evaluate surface roughness and a non-destructive vibrational test was used to evaluate dynamic bending strength during aging treatment. These methods evaluated the effect of aging treatment effectively. The aging treatment increased surface roughness and loss tangent $(\tan \delta$ ) values, but decreased dynamic modulus of elasticity $\left(E_{\mathrm{d}}\right)$ values of the panels compared to the respective initial values. The increment of surface roughness and $E_{\mathrm{d}}$ degradations observed were larger than the tan $\delta$ degradations. Surface roughness and $\tan \delta$ seemed to be relatively dependent on resin type, whereas $E_{\mathrm{d}}$ was relatively independent under the aging treatment. Overall, the medium density fiberboard bonded methylene diphenyl diisocyanate (MDF(MDI)) panel was superior among the types of panels studied under the aging treatment. There was strong correlation between average roughness $\left(R_{\mathrm{a}}\right)$ increment and $E_{\mathrm{d}}$ retention.
\end{abstract}

Keywords Particleboard - Medium density fiberboard . Accelerated aging treatment $\cdot$ Surface roughness $\cdot$ Dynamic modulus of elasticity

Shigehiko Suzuki

afssuzu@ipc.shizuoka.ac.jp

1 Faculty of Agriculture, Shizuoka University, 836-Ohya, Suruga-ku, Shizuoka 422-8529, Japan

2 Faculty of Forestry, Hasanuddin University, Perintis Kemerdekaan Km.10, Makassar 90245, Indonesia

\section{Introduction}

Properties of wood-based panels change during their service life. Therefore, panel durability over application time is an important property that needs to be evaluated. Accelerated aging tests [1-4] have been used to determine the durability of wood-based panels. Dimensional stability [5-7], bending strength [8-10], and internal bond [11-13] are parameters usually evaluated for determination of panel durability. Surface properties are less explored, and usually evaluated separately with those aforementioned parameters. The strength properties are important for meeting the requirements during use, whereas changes in surface properties should be evaluated to predict effective performance of panels during their service life.

Surface roughness, a surface property that is considered as latent, becomes notable when a panel is subjected to conditions that change its properties, such humidity. Numerous studies have reported surface roughness of wood-based panel products [14-20]. However, less information is available on surface roughness of panel as function of accelerated aging. A study exposing medium density fiberboard (MDF) to some level of relative humidity (RH) found that roughness values increased as panels were exposed to higher humidity levels ranging from 65 to 85\% [21]. However, the RH exposure was used for once until the intended equilibrium moisture content of MDF was reached. A previous study on hardboard and MDF subjected to one cycle of 50-86-50\% RH exposure [22] and found roughness instability after re-exposure to lower RH. Those RH exposures mentioned above provide insufficient information on panel surface instability that might occur owing to natural swelling and shrinkage during service life. 
Ostman [23] studied surface roughness of painted and unpainted commercial particleboard (PB) and fiberboard that were subjected to different methods of accelerated aging. The methods used were not suitable for evaluating surface roughness, because those methods employed extreme conditions that resulted in drastic degradation. The relation between surface degradation and strength degradation has been discussed; however, strength degradation and surface degradation have been studied separately. More information that is comprehensive and based on simultaneous assessment of surface and strength property degradations owing to accelerated aging tests is required.

In addition, evaluation of strength degradation is usually carried out using destructive tests. This requires plenty of specimens, and different specimens are measured on each occasion. Therefore, a mild accelerated aging method using the non-destructive bending test and the stylus technique for evaluating mechanical properties and surface roughness evaluation, respectively, is required for obtaining precise information on degradation properties.

The objective of the present study was to evaluate the changes in surface roughness and dynamic mechanical properties that occurred in MDFs and PBs, when subjected to accelerated aging treatment. In addition, we investigated the possible correlation of surface degradation with mechanical degradation.

\section{Materials and methods}

\section{Specimen preparation and accelerated aging}

Panels used in the present study were commercially manufactured PB and MDF. Two types of PB included $\mathrm{PB}$ bound with phenol formaldehyde $(\mathrm{PB}(\mathrm{PF}))$ and methylene diphenyl diisocyanate (PB(MDI)). Two types of MDF included MDF bound with melamine urea formaldehyde (MDF(MUF)) and methylene diphenyl diisocyanate (MDF(MDI)). Two panel boards in $300 \mathrm{~mm} \times 300 \mathrm{~mm}$ were selected for each panel type. The two boards were cut into ten specimens of $50 \mathrm{~mm} \times 300 \mathrm{~mm}$ and then randomly selected five specimens for each panel type. A total of twenty specimens were used for all panel types, and those were conditioned to $20{ }^{\circ} \mathrm{C}$ and $60 \% \mathrm{RH}$ for two weeks prior to the accelerated aging treatment. The initial properties of the specimens are shown in Table 1.

The accelerated aging condition (hereafter referred to as the aging treatment) consisted of fifteen cycles of exposure to high and low $\mathrm{RH}$ at a constant temperature of $60{ }^{\circ} \mathrm{C}$. The cycle started with the wet state $(90 \% \mathrm{RH}$ for $120 \mathrm{~h})$, followed by the dry state (no humidity control for $48 \mathrm{~h}$ ). Thus, each cycle lasted one week. This present study used water vapor instead of liquid water, as required by several standardized accelerated aging test [1-4].

\section{Measurement and testing}

Dimensions and weight of specimens, measured after conditioning at $20{ }^{\circ} \mathrm{C}$ and $60 \% \mathrm{RH}$, was specified as initial state of the specimens. The same specimens were evaluated for thickness swelling (TS), surface quality, and a dynamic bending test was carried out at the end of every state during each cycle of the aging treatment. For surface quality evaluation, surface profiles were measured with a stylustype profilometer (SJ-301, Mitutoyo Surftest). The tracing length was $15 \mathrm{~mm}$ with a constant speed of $0.5 \mathrm{~mm} / \mathrm{s}$. Measurements were made perpendicular to direction of the board production. Three points on the surface of the each specimen, one point was in the center surface and the other points were $20 \mathrm{~mm}$ distance from the both panel edges, were marked. Those are to ensure that the same point was measured on each occasion. Calibration of the device was performed before the measurement.

Three roughness parameters, average roughness $\left(R_{\mathrm{a}}\right)$, maximum height roughness $\left(R_{\mathrm{z}}\right)$, and ten points mean roughness $\left(R_{\mathrm{zJIS}}\right)$, which are commonly used to evaluate surface characteristic of wood and wood-based panels, were calculated to determine the degradation indicated by surface roughness over the humidity change exposure. The definitions of these three parameters are available in JIS B 0601-2001 [24]. Increasing rate of those surface roughness parameters were determined by Eq. (1).

Roughness increasing rate $(\%)$
$=\frac{\text { Value after aging }- \text { value before aging }}{\text { Value before aging }} \times 100$

In addition to surface roughness measurements, microstructure of the panel surfaces was examined using scanning electron microscope (JSM-6510LV, Joel). Unaged specimens and specimen after completion of aging treatment were inspected. Moreover, the term surface degradation in this study is limited in the increment of surface structure change compared to its initial surface condition.

Evaluation of mechanical properties was performed simultaneously with surface roughness measurements. Dynamic modulus of elasticity $\left(E_{\mathrm{d}}\right)$ values of $(2,0)$ vibration mode and loss tangent $(\tan \delta)$ were determined with a non-destructive vibration method using sound level meter (LA-1410, Ono Sokki) with fast Fourier transform (FFT) analyzer (CF-7200, Ono Sokki). A microphone was placed above the specimen end and a small hammer stroked on the opposite end. The vibration signal, which was obtained by a microphone, was converted into a power spectrum by FFT analyzer. The $E_{\mathrm{d}}$ was calculated from the peak 
Table 1 Initial properties of panel specimens in aging treatment

\begin{tabular}{llllrr}
\hline Panel type & Adhesive & Symbol & Thickness $^{\mathrm{a}}(\mathrm{mm})$ & Density $^{\mathrm{b}}\left(\mathrm{g} \mathrm{cm}^{-3}\right)$ & $E_{\mathrm{d}}^{\mathrm{c}}\left(\mathrm{GPa}^{2}\right)$ \\
\hline Particleboard (PB) & PF $^{\mathrm{d}}$ & PB(PF) & $12.0 \pm 0.03$ & $0.75 \pm 0.01$ & $4.5 \pm 0.23$ \\
& MDI $^{\mathrm{e}}$ & PB(MDI) & $12.0 \pm 0.09$ & $0.79 \pm 0.02$ & $5.3 \pm 0.30$ \\
Medium density fibreboard (MDF) & MUF $^{\mathrm{f}}$ & MDF(MUF) & $12.1 \pm 0.08$ & $0.74 \pm 0.01$ & $5.1 \pm 0.08$ \\
& MDI & MDF(MDI) & $9.0 \pm 0.03$ & $0.72 \pm 0.02$ & $4.0 \pm 0.13$
\end{tabular}

${ }^{a}$ Thickness is given as average value \pm standard deviation

${ }^{\mathrm{b}}$ Density is given as average value \pm standard deviation

c Dynamic modulus of elasticity, given as average value \pm standard deviation

d Phenol formaldehyde

e Methylene diphenyl diisocyanate

${ }^{\mathrm{f}}$ Melamine urea formaldehyde

resonance frequency by Eq. (2) and tan $\delta$ was calculated from the amplitude of the resonance curve by Eq. (3).

$E_{\mathrm{d}}=\frac{48 \pi^{2} \rho L^{4} f^{2}}{500.6 t^{2}}(\mathrm{GPa})$,

$\tan \delta=\frac{\lambda}{\pi}$,

where $\rho$ is density $\left(\mathrm{g} \mathrm{cm}^{-3}\right), L$ is the length ( $\left.\mathrm{mm}\right), f$ is the frequency of the resonance peak $(\mathrm{Hz}), t$ is the thickness $(\mathrm{mm})$, and $\lambda$ is the logarithmic decrement of the resonance amplitude which calculated by Hilbert transformation. Authors' previous study [25] determined the $E_{\mathrm{d}}$ based on the initial thickness and density values, whereas in this present study, based on the thickness and density values at the test. Panel $E_{\mathrm{d}}$ degradation upon the aging treatment was expressed by $E_{\mathrm{d}}$ residual value and calculated using Eq. (4).

$E_{\mathrm{d}}$ retention $(\%)=\frac{E_{\mathrm{d}} \text { value at test }}{E_{\mathrm{d}} \text { value at initial }} \times 100$

\section{Results and discussion}

\section{Effect of the aging treatment on surface quality}

Surface profile was recorded before, during and after the aging treatment. Surface profile provides a prompt visual view of panel surface changes. Surface structure changed after the aging treatment, which was evident from the longer raised and lowered strips than those visible before the aging treatment (Fig. 1). However, the figure seems quite difficult to be used for comparing the changes between different types of panels. Therefore, quantitatively surface roughness before and after the aging treatment was identified. Three roughness parameters, $R_{\mathrm{a}}, R_{\mathrm{z}}$, and $R_{\mathrm{zJIS}}$, reported as the mean of fifteen different profiles for each panel type, increased with the aging treatment (Table 2). The increasing rate in $R_{\mathrm{a}}$ appeared to be larger than that of $R_{\mathrm{z}}$ and $R_{\mathrm{zJIS}}$. MDF(MUF) showed the largest increasing rate with the aging treatment $(76 \%)$, followed by $\mathrm{PB}(\mathrm{PF})$ (74\%), PB(MDI) (62\%), and MDF(MDI) (22\%). The order of increasing rate in $R_{\mathrm{z}}$ was the same as $R_{\mathrm{a}}$, whereas the largest increasing rate in $R_{\text {zJIS }}$ was found in $\mathrm{PB}(\mathrm{PF})(44 \%)$, followed by $\mathrm{MDF}(\mathrm{MUF})$ (40\%), $\mathrm{PB}(\mathrm{MDI})(27 \%)$, and MDF(MDI) (9\%). Surface changes obtained were considered to be very small compared to surface changes under vacuum pressure-soak-dry (VPSD) aging reached 325\% for PB phenolic resin and $222 \%$ for fiberboard phenolic resin [23].

Even though PB(MDI) had the coarsest surface initially, it showed lower increasing rate in surface roughness parameters than the two types of amino-based panels, (i.e., $\mathrm{MDF}(\mathrm{MUF})$ and $\mathrm{PB}(\mathrm{PF})$ ). In contrast, $\mathrm{MDF}(\mathrm{MUF})$ with the smoothest surface initially, became rough after the aging treatment, indicating heavy degradation. However, MDF(MDI) exhibited the least increasing rate in surface roughness parameters. In addition, the change in surface roughness of the panels depends on the adhesive type.

The surface changes occurred in panel specimens owing to moisture content variation by the aging treatment. A change during the aging treatment is valuable information for understanding the progress of degradation over time and predicting aging resistance. Since the panels did not degrade drastically by the aging treatment, the stylus technique could trace the surface changes during the treatment. $R_{\mathrm{a}}$ increased with the number of cycles, indicating degradation of the panel surface (Fig. 2). Large degradation was evident in the first cycle, followed by relatively gradual degradation. Degradation appeared to progress in $\mathrm{PB}(\mathrm{PF}), \mathrm{PB}(\mathrm{MDI})$, and $\mathrm{MDF}(\mathrm{MUF})$ panels, whereas $R_{\mathrm{a}}$ remained constant in the MDF(MDI) panel. 

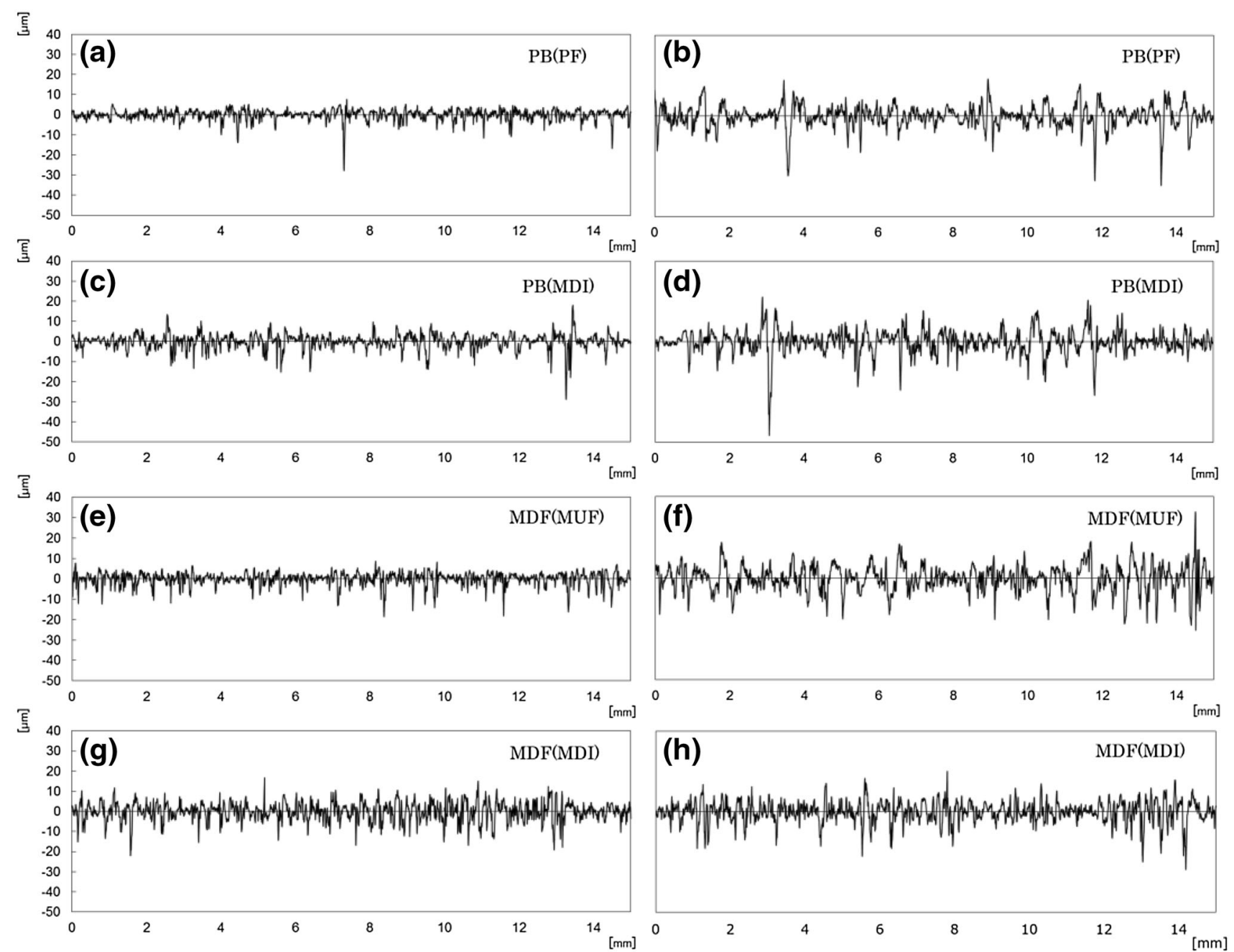

Fig. 1 Typical vicinal surface profile before (left side) and after (right side) the aging treatment; a, b PB(PF), c, d PB(MDI), e, f MDF(MUF), and $\mathbf{g}, \mathbf{h}$ MDF(MDI)

Table 2 Surface roughness parameters of the panels before and after the aging treatment

\begin{tabular}{|c|c|c|c|c|c|c|c|c|c|}
\hline \multirow[t]{2}{*}{ Panel type } & \multicolumn{2}{|l|}{$R_{\mathrm{a}}^{\mathrm{e}}(\mu \mathrm{m})$} & \multicolumn{2}{|l|}{$R_{\mathrm{z}}^{\mathrm{f}}(\mu \mathrm{m})$} & \multicolumn{2}{|l|}{$R_{\mathrm{zJIS}}^{\mathrm{g}}(\mu \mathrm{m})$} & \multicolumn{3}{|c|}{ Increasing rate $(\%)$} \\
\hline & Before aging & After aging & Before aging & After aging & Before aging & After aging & $R_{\mathrm{a}}$ & $R_{\mathrm{z}}$ & $R_{\mathrm{zJIS}}$ \\
\hline $\mathrm{PB}(\mathrm{PF})^{\mathrm{a}}$ & $2.4 \pm 0.4$ & $4.2 \pm 0.8$ & $39.8 \pm 14.5$ & $54.0 \pm 20.5$ & $28.9 \pm 6.8$ & $41.6 \pm 9.0$ & 74 & 36 & 44 \\
\hline $\mathrm{PB}(\mathrm{MDI})^{\mathrm{b}}$ & $3.0 \pm 0.7$ & $4.8 \pm 0.6$ & $59.1 \pm 11.4$ & $68.9 \pm 8.8$ & $40.9 \pm 8.2$ & $51.9 \pm 7.1$ & 62 & 17 & 27 \\
\hline $\operatorname{MDF}(\mathrm{MUF})^{\mathrm{c}}$ & $2.2 \pm 0.3$ & $3.9 \pm 0.6$ & $34.7 \pm 12.7$ & $48.4 \pm 9.8$ & $26.0 \pm 3.9$ & $36.5 \pm 5.2$ & 76 & 40 & 40 \\
\hline $\operatorname{MDF}(\mathrm{MDI})^{\mathrm{d}}$ & $2.3 \pm 0.5$ & $2.8 \pm 0.4$ & $32.2 \pm 6.3$ & $35.1 \pm 5.9$ & $26.0 \pm 4.6$ & $28.4 \pm 4.4$ & 22 & 9 & 9 \\
\hline
\end{tabular}

Values given as mean \pm standard deviation

a Particleboard bound with phenol formaldehyde

b Particleboard bound methylene diphenyl diisocyanate

c Medium density fiberboard bound with melamine urea formaldehyde

${ }^{\mathrm{d}}$ Medium density fiberboard bound with methylene diphenyl diisocyanate

e Average roughness

${ }^{\mathrm{f}}$ Maximum height roughness

g Ten points mean roughness 

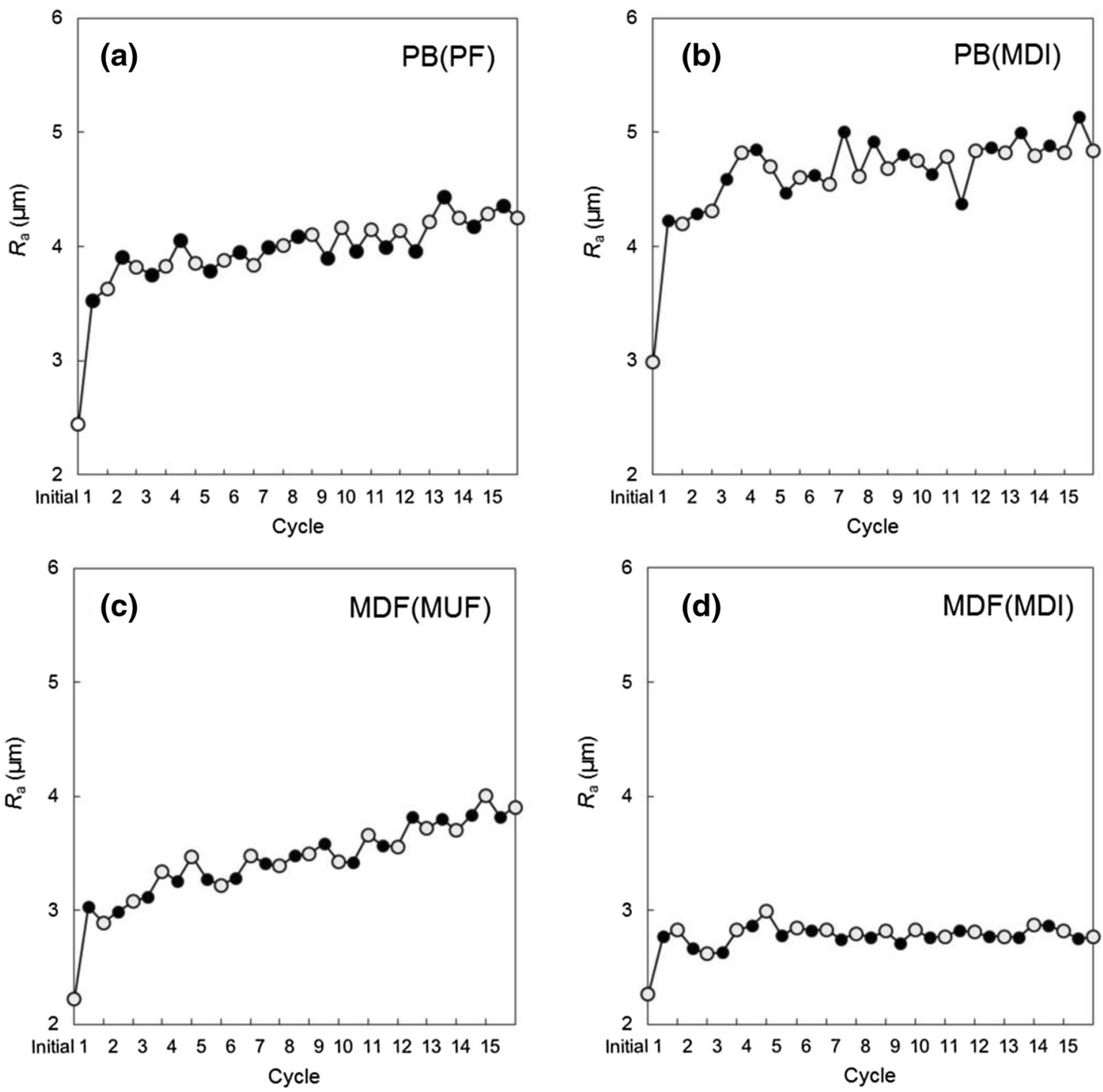

Fig. 2 Average roughness $\left(R_{\mathrm{a}}\right)$ changes during aging treatment; a $\mathrm{PB}(\mathrm{PF}), \mathbf{b} \mathrm{PB}(\mathrm{MDI}), \mathbf{c} \mathrm{MDF}(\mathrm{MUF})$, and d MDF(MDI)

Surface roughness degradation is associated with dimensional changes owing to swelling-shrinkage phase during aging treatments [26]. Each panel had different extent and course of change in TS following the aging treatment (Fig. 3). TS of PBs increased gradually at the dry state of each cycle, whereas MDFs showed little change in the dry states. Unlike TS change, which increased and decreased intermittently at wet and dry states, respectively, $R_{\mathrm{a}}$ did not show a similar trend in each cycle. Changes in surface roughness owing to aging treatment were irreversible. Increasing surface roughness appeared consistent with increasing TS in PB panels, whereas it did not in MDF panels. Even though MDF(MUF) experienced little change TS, swelling-shrinkage could loosen and raised some individual fibers on the surface. It did not affect the overall
TS but fiber-pop affected the scanning track of the stylus. Comparing the courses of surface degradation owing to the aging treatment revealed that MDF(MDI) had the greatest surface stability among the panel types compared. This might be attributed to the combination of MDI resin used and low density of the panel (lower compaction ratio) could produce high surface stability. High density particleboard surface are not as stable as low density when subjected to RH changes [14].

Micrographs of the unaged two types of MDF panel surfaces appeared almost similar (Fig. 4), consistent with the quantitative measures of surface roughness values. However, the surface structure became uneven after the aging treatment, with more cracks and raised fibers in MDF(MUF) than in MDF(MDI) panel surface. 

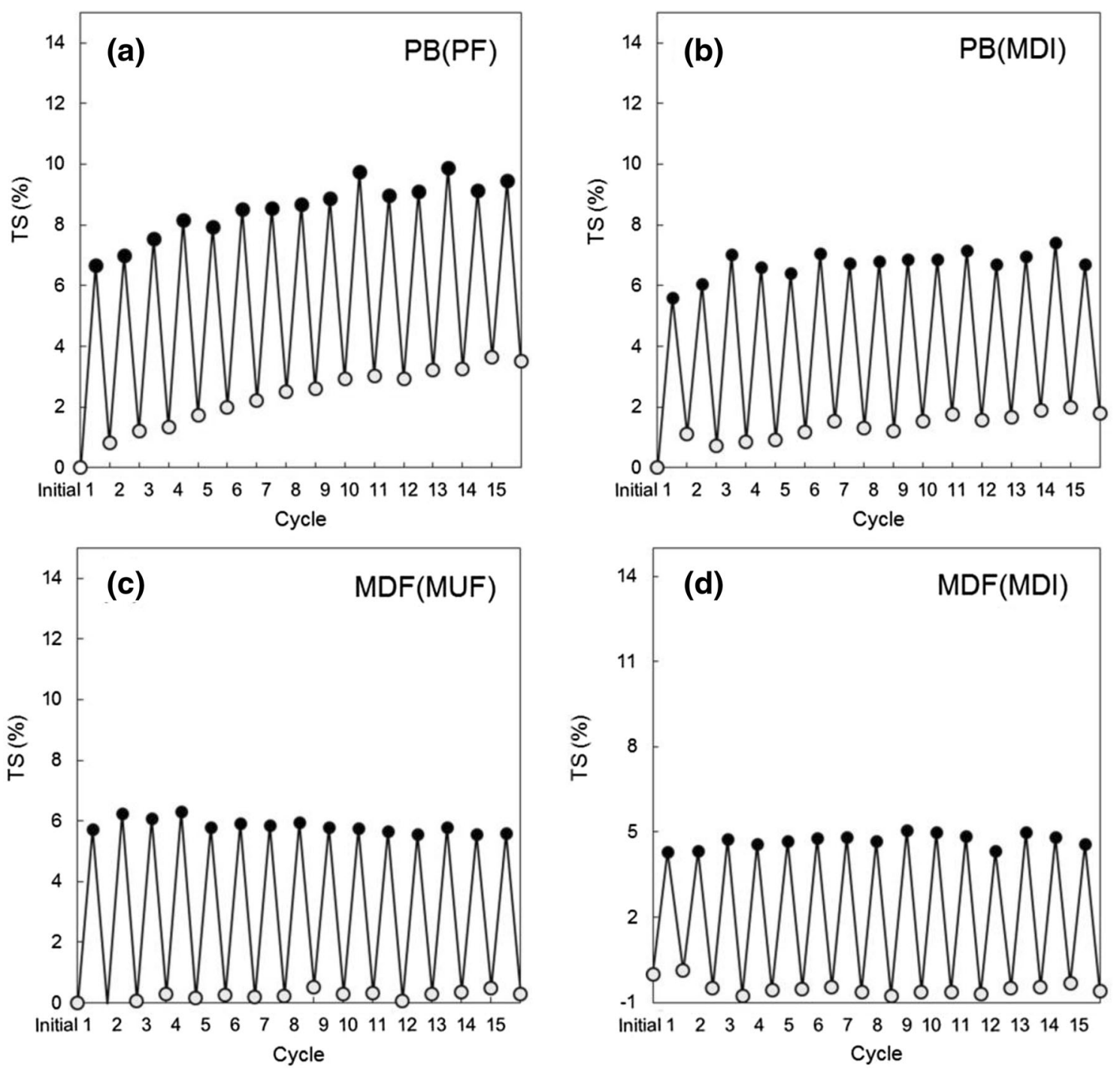

Fig. 3 Thickness swelling (TS) during aging treatment; a PB(PF), b PB(MDI), c MDF(MUF), and d MDF(MDI)

\section{Effect of aging treatment on mechanical properties}

Dynamic modulus of elasticity $\left(E_{d}\right)$

The $E_{\mathrm{d}}$ value before the aging treatment ranged from approximately 4.0 to $5.3 \mathrm{GPa}$ (Fig. 5). In contrast, after the aging treatment, the $E_{\mathrm{d}}$ value ranged from 2.1 to $3.3 \mathrm{GPa}$ and 2.7 to $4.0 \mathrm{GPa}$ in the wet and dry states, respectively. The results revealed that the aging treatment reduced $E_{\mathrm{d}}$ values of all the panels. Among the panel types compared, $\mathrm{PB}(\mathrm{PF})$ had the lowest $E_{\mathrm{d}}$ value after the aging treatment. Observing $E_{\mathrm{d}}$ changes using non-destructive test could follow the progress degradation owing to the aging treatment. $E_{\mathrm{d}}$ retention of the panels, which showed the progress during the treatment, revealed that unlike surface degradation, the aging treatment had similar effects on $E_{\mathrm{d}}$ at each wet and dry state in every cycle (Fig. 6).
Degradation increased with increasing number of cycles. We observed that rapid degradation occurred at the first cycle, followed by slow decrease in rate of degradation at successive cycles. $E_{\mathrm{d}}$ retention of $\mathrm{PB}(\mathrm{PF})$, $\mathrm{PB}(\mathrm{MDI})$ and $\mathrm{MDF}(\mathrm{MUF})$ decreased exponentially with increasing number of cycles, whereas that in MDF(MDI) hardly decreased. $\mathrm{PB}(\mathrm{PF})$ showed the least $E_{\mathrm{d}}$ retention $(59 \%)$ after the completion of the aging treatment, followed by MDF(MUF) (75\%), PB(MDI) (75\%), and MDF(MDI) (93\%). Generally, amino-based panels, in which PF is known as a less durable resin than MDI, showed larger $E_{\mathrm{d}}$ loss than that in MDI-bonded panels. However, it was revealed that $\mathrm{PB}(\mathrm{MDI})$ and MDF(MUF) had similar decreasing trend and equal values in $E_{\mathrm{d}}$ retention (Fig. 6). It could be interpreted that both panels have similar resistance or performance under this aging treatment. Therefore, resin type was not major factor for 

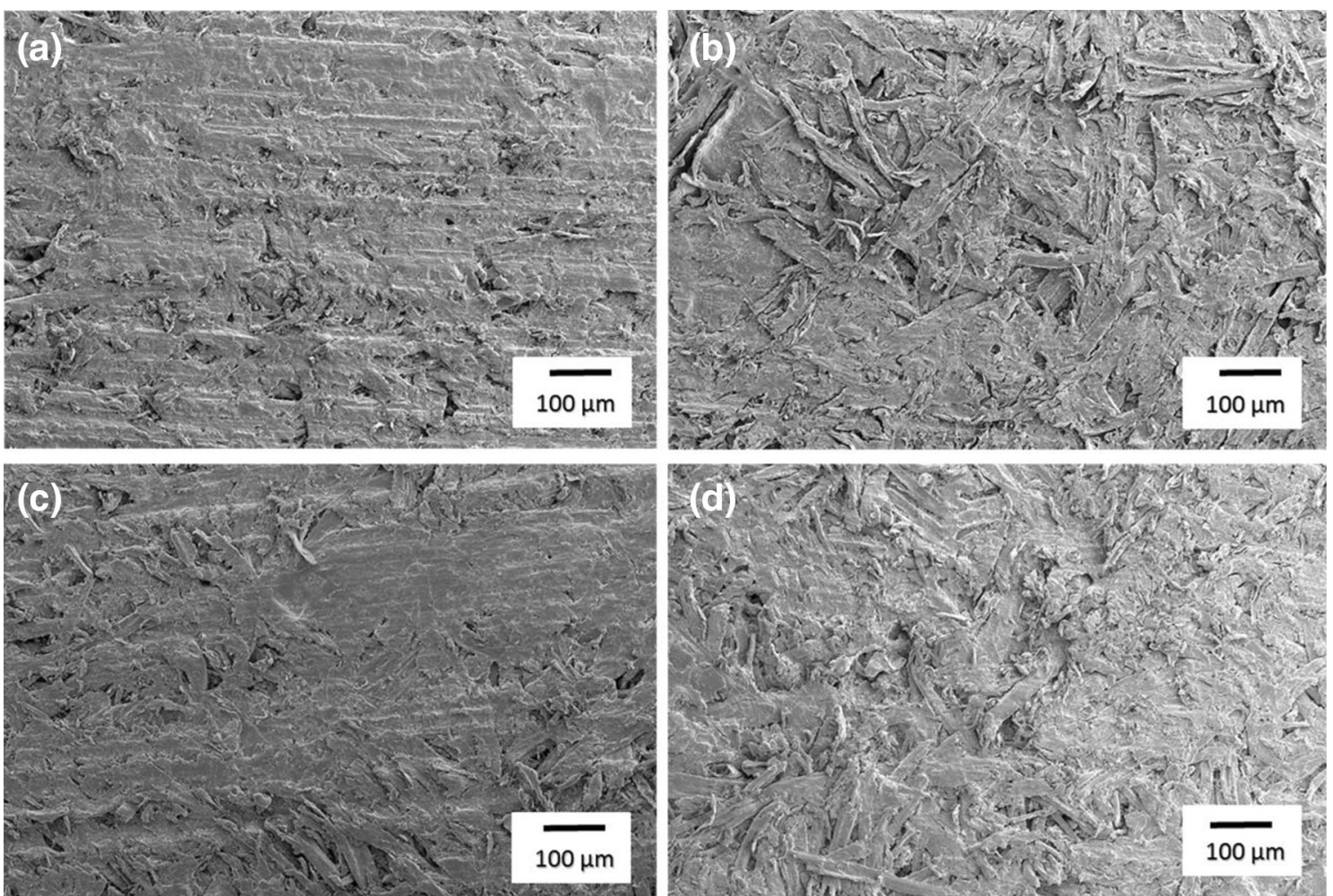

Fig. 4 Micrographs $(\times 100)$ of the surface of unaged MDF(MUF) (a), aged MDF(MUF) (b), unaged MDF(MDI) (c), and aged MDF(MDI) (d)

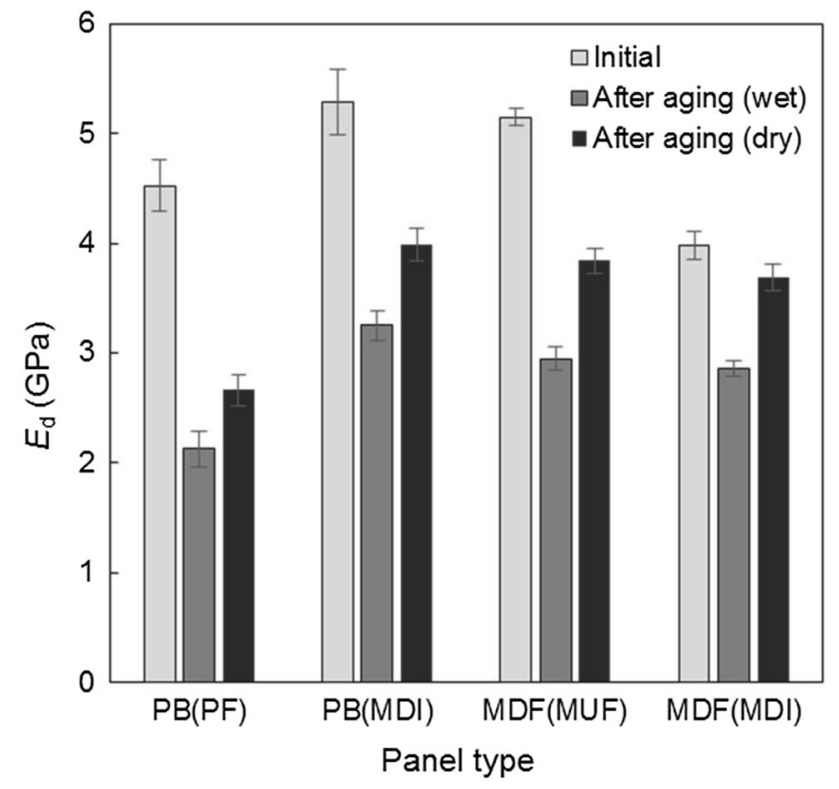

Fig. 5 Average $E_{\mathrm{d}}$ values of panels before and after the aging treatment

$E_{\mathrm{d}}$ degradation as it is found in surface degradation. Interaction factor between resin type and panel constituent might contribute to the $E_{\mathrm{d}}$ degradation as well.

\section{Loss tangent (tan $\delta)$}

The $\tan \delta$ values after the aging treatment did not show marked changes compared to the initial state, except in MDF(MUF). Slight increase in $\tan \delta$ with increasing number of cycles was observed during the aging treatment in the wet state of $\mathrm{PB}(\mathrm{PF})$ and $\mathrm{MDF}(\mathrm{MUF})$, whereas $\mathrm{PB}(\mathrm{MDI})$ and $\mathrm{MDF}(\mathrm{MDI})$ showed hardly changed (Fig. 7). Moreover, the change in $\tan \delta$ between the dry and wet states of the two amino-based panels was larger than that in the two MDI-bound panels. This indicates that the change in $\tan \delta$ is relatively dependent of resin type and MDIbonded panels were able to maintain their formation against humidity exposure. Furthermore, the change in tan $\delta$ was much less than that in surface degradation and $E_{\mathrm{d}}$ degradation.

\section{Correlation of surface and mechanical degradation}

To compare degradations more easily, a ranking was made using value of the changes after treatment cycles, based on their respective initial values. The panel types studied could be arranged in decreasing order of surface degradation as follows: $\mathrm{MDF}(\mathrm{MUF}), \mathrm{PB}(\mathrm{PF}), \mathrm{PB}(\mathrm{MDI})$, and $\mathrm{MDF}(\mathrm{MDI})$. In contrast, $E_{\mathrm{d}}$ and $\tan \delta$ degradation were similar in $\mathrm{PB}(\mathrm{PF}), \quad \mathrm{MDF}(\mathrm{MUF}), \quad \mathrm{PB}(\mathrm{MDI}), \quad$ and 

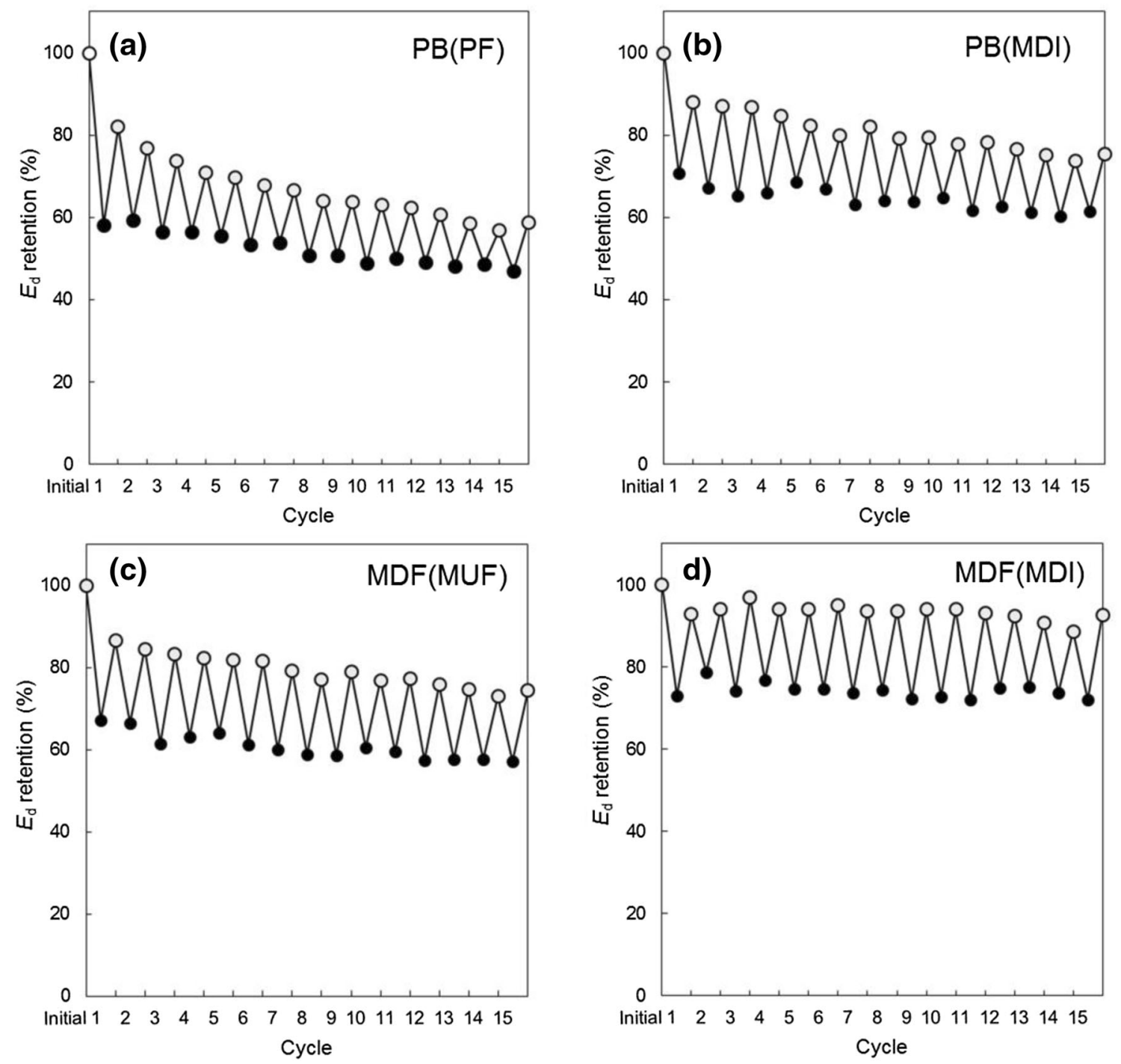

Fig. $6 E_{\mathrm{d}}$ retention during aging treatment; a $\mathrm{PB}(\mathrm{PF}), \mathbf{b} \mathrm{PB}(\mathrm{MDI}), \mathbf{c} \mathrm{MDF}(\mathrm{MUF})$, and $\mathbf{d} \mathrm{MDF}(\mathrm{MDI})$. White circles are values in dry state and black circles are values in wet state

$\mathrm{MDF}(\mathrm{MDI})$. However, $E_{\mathrm{d}}$ loss of $\mathrm{PB}(\mathrm{PF})$ was much more marked than surface degradation, in contrast to the observed trends in MDF(MUF). Nevertheless, the two panels bound with MDI resin exhibited the same rank for those surface and mechanical degradations.

To evaluate the feasibility of predicting total degradation, correlation between surface degradation and mechanical degradations was determined. Changes of $R_{\mathrm{a}}$, $E_{\mathrm{d}}$, and $\tan \delta$ values at each dry state during the aging treatment were analyzed. Generally, stronger correlation existed between $R_{\mathrm{a}}$ and $E_{\mathrm{d}}$ (Fig. 8a) as higher correlation coefficient value than that in $R_{\mathrm{a}}$ and $\tan \delta$ (Fig. 8b). $R_{\mathrm{a}}$ and $E_{\mathrm{d}}$ of $\mathrm{PB}(\mathrm{MDI})$ and $\mathrm{MDF}(\mathrm{MUF})$ had higher correlation ( 0.81 and 0.91 , respectively). These correlations mean that the change at successive cycle in surface roughness might be used to predict the change in dynamic modulus of elasticity. In case of correlation between $R_{\mathrm{a}}$ and $\tan \delta$, only $\mathrm{PB}(\mathrm{PF})$ appeared to have high correlation (0.82) compared to the other panels. The reasons of the low correlation of $R_{\mathrm{a}}$ and $\tan \delta$ rather than $R_{\mathrm{a}}$ and $E_{\mathrm{d}}$ remain unclear at present. However, in vibration method, $E_{\mathrm{d}}$ relates to the apparent thickness of the board, whereas $\tan \delta$ corresponds to the property of in-plane board. Presumably, this is a consequence of that.

\section{Conclusion}

Surface roughness and dynamic mechanical properties of commercial PB panels and MDF panels subjected to the aging treatment of repetitive cycles of RH fluctuation were evaluated. The stylus technique and a non-destructive 

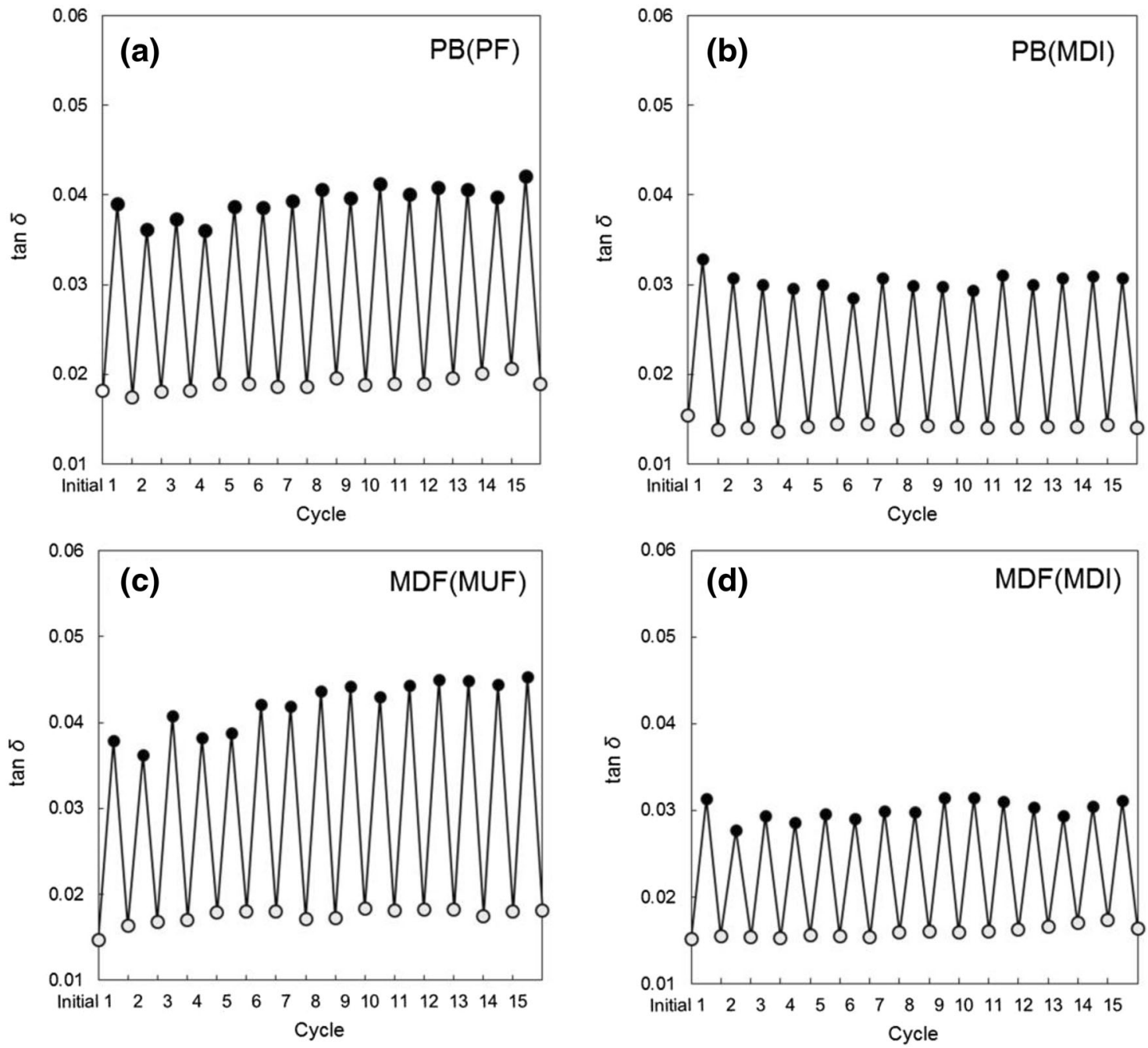

Fig. 7 Measurements of $\tan \delta$ during aging treatment; a $\mathrm{PB}(\mathrm{PF}), \mathbf{b} \mathrm{PB}(\mathrm{MDI}), \mathbf{c} \mathrm{MDF}(\mathrm{MUF})$, and $\mathbf{d} \mathrm{MDF}(\mathrm{MDI})$. White circles are values in dry state and black circles are values in wet state

Fig. 8 Changes in the relation between surface roughness and mechanical properties of panels at dry condition. a $R_{\mathrm{a}}$ and $E_{\mathrm{d}}$, and $\mathbf{b} R_{\mathrm{a}}$ and $\tan \delta$
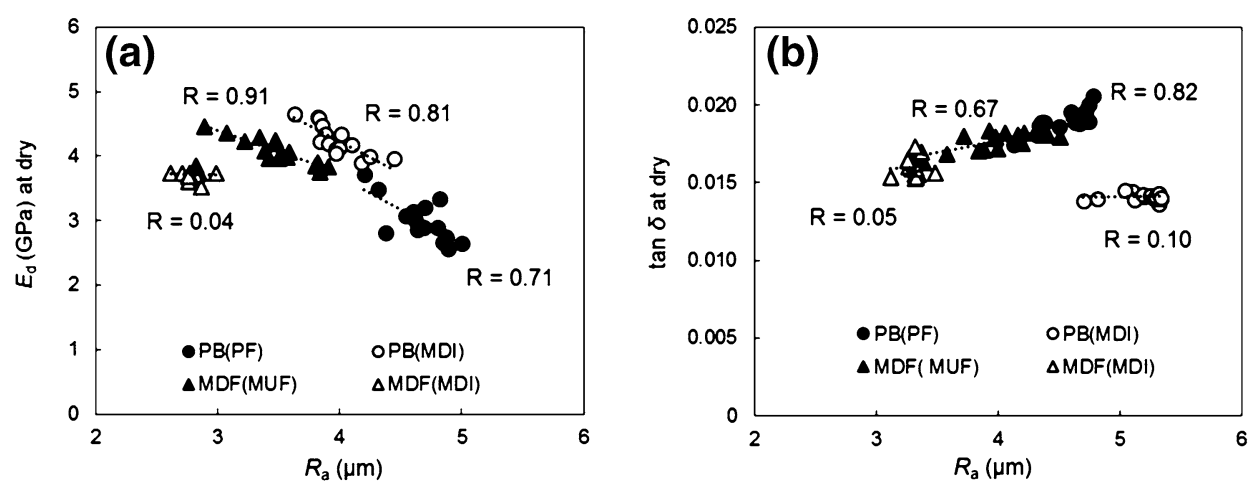

vibrational test were effective in following degradation during the aging treatment. The aging treatment increased surface roughness and $\tan \delta$ values, but decreased $E_{\mathrm{d}}$ values of the panels compared to their respective initial values. The $R_{\mathrm{a}}$ increment and $E_{\mathrm{d}}$ retention observed were more marked than $\tan \delta$ increment. The degradation behavior during aging treatment appeared to be different among the panels. Surface roughness and $\tan \delta$ seemed to be relatively dependent on resin type, whereas $E_{\mathrm{d}}$ was relatively independent. Overall, $\mathrm{MDF}(\mathrm{MDI})$ was superior in both surface 
roughness and dynamic mechanical properties. There was strong correlation between $R_{\mathrm{a}}$ increment and $E_{\mathrm{d}}$ retention. Surface roughness change might be used to predict dynamic modulus of elasticity, particularly in MDF(MUF) panel.

\section{References}

1. ASTM-D1037 (1999) Standard test method for evaluating properties of wood-base fiber and particle panel materials. American Society for Testing and Materials (ASTM), West Conshohocken, p 19

2. EN321 (1993) Fiberboards: cyclic testing humid condition. European Standard, Brussels

3. APA PRP 108 (1994) Performance standards and policies for structural-use panels. APA-The Engineered Wood Association, Tacoma, p 33

4. JIS A-5908 (2003) Particleboard. JIS standard specification, Japanese Standards Association, Tokyo, p 14

5. Pugel AD, Price EW, Hse CY (1990) Composites from southern pine juvenile wood. Part 2. Durability and dimensional stability. For Prod J 40(3):57-61

6. Moya L, Tze WTY, Winandy JE (2009) The effect of cyclic relative humidity changes on moisture content and thickness swelling behavior of oriented strandboard. Wood Fiber Sci 41(4):447-460

7. Kojima Y, Norita H, Suzuki S (2009) Evaluating the durability of wood-based panels using thickness swelling results from accelerated aging treatments. For Prod J 59(5):35-41

8. Hann RA, Black JM, Blomquist RF (1963) How durable is particleboard? Part II. For Prod J 13(5):169-174

9. Kojima Y, Suzuki S (2011) Evaluation of wood-based panel durability using bending properties after accelerated aging treatments. J Wood Sci 57:126-133

10. Korai H, Saotome H, Ohmi M (2014) Effects of water soaking and outdoor exposure on modulus of rupture and internal bond strength of particleboard. J Wood Sci 60:127-133

11. Kajita H, Mukudai J, Yano H (1991) Durability evaluation of particleboards by accelerated aging tests. Wood Sci Technol 25:239-249

12. Kojima Y, Suzuki S (2011) Evaluation of wood-based panel durability using bending internal bond strength results from accelerated aging treatments. J Wood Sci 57:7-13
13. Korai H, Kojima Y, Suzuki S (2015) Bending strength and internal bond strength of wood-based boards subjected to various exposure conditions. J Wood Sci 61:500-509

14. Hiziroglu S, Suchland O (1993) Linear expansion and surface stability of particleboard. For Prod J 43(4):31-34

15. Hiziroglu S, Jarusombuti S, Fueangvivat V (2004) Surface characteristic of wood composite manufactured in Thailand. Build Environ 39:1359-1364

16. Hiziroglu S, Kosonkorn P (2006) Evaluation of surface roughness of Thai medium density fiberboard (MDF). Build Environ 41:527-533

17. Hiziroglu S, Suzuki S (2007) Evaluation of surface roughness of commercially manufactured particleboard and medium density fiberboard in Japan. J Mater Process Technol 184:436-440

18. Kilic M, Burdurlu E, Aslan S, Altun S, Tumerdem O (2009) The effect of surface roughness on tensile strength of medium density fiberboard (MDF) overlaid with polyvinyl chloride (PVC). Mater Des 30:4580-4583

19. Tabarsa T, Ashori A, Gholamzadeh M (2011) Evaluation of surface roughness and mechanical properties of particleboard panels made from bagasse. Compos Part B 42:1330-1335

20. Baharoglu M, Nemli G, Sari B, Bardak S, Ayrilmis N (2012) The influence of moisture content of raw material on the physical and mechanical properties, surface roughness, wettability, and formaldehyde emission of particleboard composite. Compos Part B 43:2448-2451

21. Ozdemir T, Hiziroglu S, Malkocoglu A (2009) Influence of relative humidity on surface quality and adhesion streght of coated medium density fiberboard (MDF) panels. Mater Des 30:2543-2546

22. Hiziroglu S (1996) Surface roughness analysis of wood composites: a stylus method. For Prod J 46(7/8):67-72

23. Ostman BAL (1983) Surface roughness of wood-based panels after aging. For Prod J 33(7/8):35-42

24. JIS B 0601 (2001) Geometrical product specifications (GPS)surface texture: profile method-terms, definitions and surface texture parameters. JIS standard specification, Japanese Standards Association, Tokyo

25. Saad S, Kobori H, Kojima Y, Suzuki S (2016) Performance evaluation of wood-based panels under a mild accelerated aging treatment. J Wood Sci 62:324-331

26. Suchsland O (1973) Hygroscopic thickness swelling and related properties of selected commercial particleboard. For Prod J 23(7):26-30 